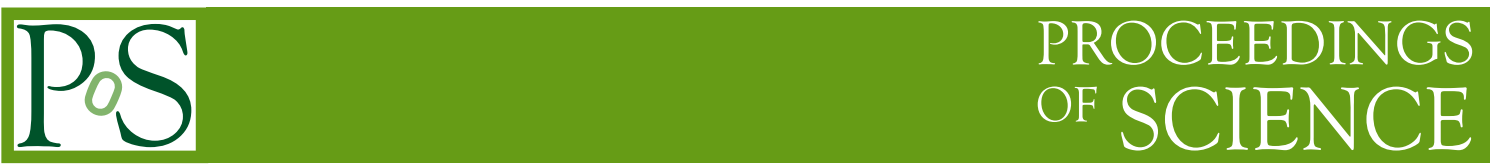

\title{
ATLAS Level-1 Endcap Muon Trigger for Run 3
}

\author{
Hiroaki Hibi * on behalf of the ATLAS Collaboration \\ Kobe University \\ E-mail: hiroaki.hibiecern.ch
}

The LHC is expected to increase its center-of-mass energy to $14 \mathrm{TeV}$ and an instantaneous luminosity to $2.4 \times 10^{34} \mathrm{~cm}^{-2} \mathrm{~s}^{-1}$ for Run 3 scheduled from 2021 to 2023 . In order to cope with the high event rate, an upgrade of the ATLAS trigger system is required. The level-1 endcap muon trigger system identifies muons with high transverse momentum measured by a fast muon trigger detector, TGC. In the ongoing upgrades for Run 3, new detectors called the New-Small-Wheel (NSW) and RPC BIS78 will be installed in the inner station region for the endcap muon trigger. Track information from the NSW and RPC BIS78 can improve the performance of the level-1 endcap muon trigger system significantly due to their good resolutions. In order to handle data from the TGC and new detectors, some new electronics have been developed, including the trigger processor board known as Sector Logic (SL). The SL board has a modern FPGA with huge resource, making it possible to implement algorithms for trigger decisions. A charge identification algorithm has been developed newly and the accuracy is estimated by simulation. Also, a trigger algorithm with NSW has been developed to reject fake triggers and improves the transverse momentum resolution. The trigger rate was estimated by real data in Run 2 .

XXIX International Symposium on Lepton Photon Interactions at High Energies - LeptonPhoton2019 August 5-10, 2019

Toronto, Canada

${ }^{*}$ Speaker. 


\section{Level-1 endcap muon trigger}

The level-1 endcap muon trigger of the ATLAS experiment [1] is a hardware trigger using Big Wheel of Thin Gap Chamber (TGC-BW) (as shown in Figure 1) used to identify muons in $1.05 \leq|\eta| \leq 2.40$ and whole $\phi$. A muon transverse momentum $\left(p_{\mathrm{T}}\right)$ is defined by measurement of curvature in the magnetic field $(\Delta R, \Delta \phi)$. Since a latency for the $p_{\mathrm{T}}$ calculation is limited, predefined Look-Up-Tables (LUTs), as shown in Figure 1, are used. Beam-induced backgrounds, primarily originating from the endcap toroid or shielding, are a significant source of fake triggers. A coincidence between TGC-BW and detectors in the inner station (inner coincidence) is the key to suppress the fake triggers. The endcap muon system is upgraded with new muon detectors, the New Small Wheel (NSW) [2] and RPC BIS78 [3][4], to suppress the fake triggers from Run 3 (2021-2023)[5]. NSW and RPC BIS78 will be installed as shown in Figure 1. A new trigger processor board (Sector Logic; SL) has a sufficient number of inputs and outputs to cope with the large amount of information from the detectors in the inner station. It is able to implement more complex and high performance algorithms since the FPGA resource is increased by a factor of 20 for Run 3.
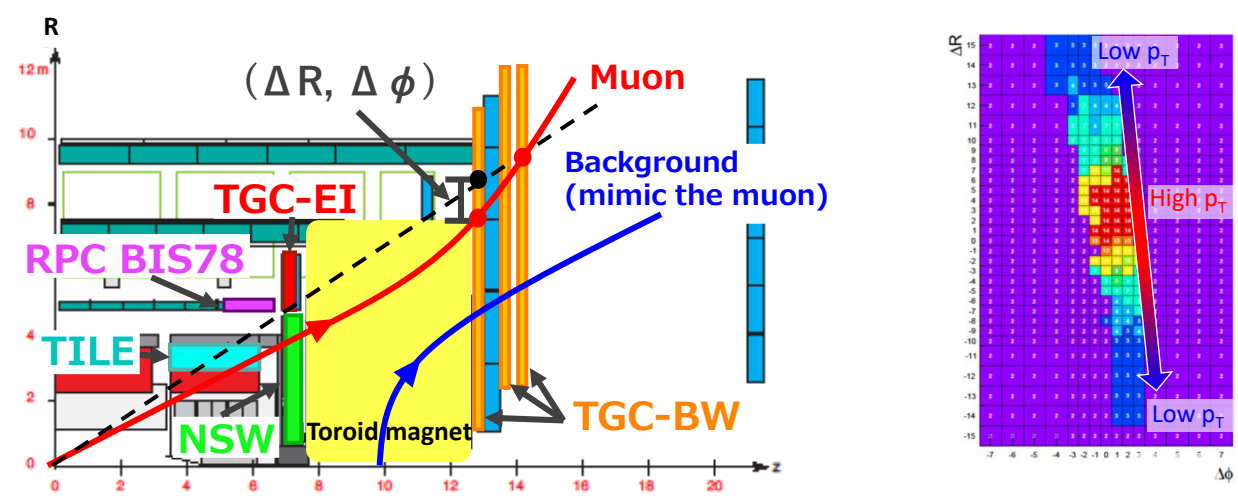

Figure 1: The schematic view of the muon spectrometer (left) and an example of the LUTs for the $p_{\mathrm{T}}$ calculation (right). TGC-EI, TILE, NSW and RPC BIS78 are inner station detectors used for inner coincidence.

\section{Improvement of muon charge identification}

The charge information is useful for development of some dedicated triggers, for example, a di-muon trigger for the decay $B_{s}^{0} \rightarrow \mu^{+} \mu^{-}$, which is a sensitive probe for physics beyond the Standard Model. The di-muons trigger generates a lot of fake contribution from accidental coincidences. The requirement of the opposite charges to the di-muon can suppress the fake triggers significantly. A good accuracy on the charge identification is available by making full use of $\Delta R-\Delta \phi$ information measured by TGC-BW. Figure 2 shows the efficiency for the level-1 muon transverse momentum trigger with a threshold of $4 \mathrm{GeV}\left(\mathrm{L} 1 \_\mathrm{MU} 4\right)$, and the efficiency with correctly identified charge using LUTs for charge identification. The identification accuracy for single muon is more than $98 \%$ in $p_{\mathrm{T}}<30 \mathrm{GeV}$. 


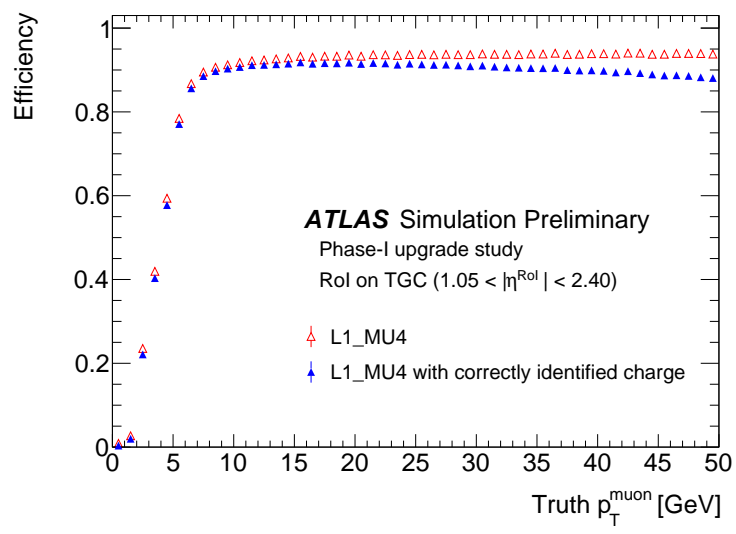

Figure 2: Charge identification performance for the L1_MU4 [6].

\section{TGC-BW and NSW coincidence}

The correlations in hit position and angle between TGC-BW and NSW are useful to suppress the fake triggers and improve the $p_{\mathrm{T}}$ resolution. The $p_{\mathrm{T}}$ is calculated by LUTs which are defined by hit maps of the position and angle generated by simulation. Figure 3 shows the number of muon candidates in the level-1 muon transverse momentum trigger with a threshold of 20 $\mathrm{GeV}\left(\mathrm{L} 1 \_\mathrm{MU} 20\right)$ as a function of $p_{\mathrm{T}}$. The candidates below the $p_{\mathrm{T}}$ threshold are suppressed by the coincidence. Figure 4 shows the rate reduction of L1_MU20 estimated using Run 2 data and resuts of single muon simulation study. In the Run 2 data, the muon segments from Monitored Drift Tube, which is a detector located in the region where NSW and RPC BIS78 will be installed, are used insted of NSW and RPC BIS78. As a result, more than $90 \%$ of the fake triggers are rejected and approximately $45 \%$ rate reduction is expected.

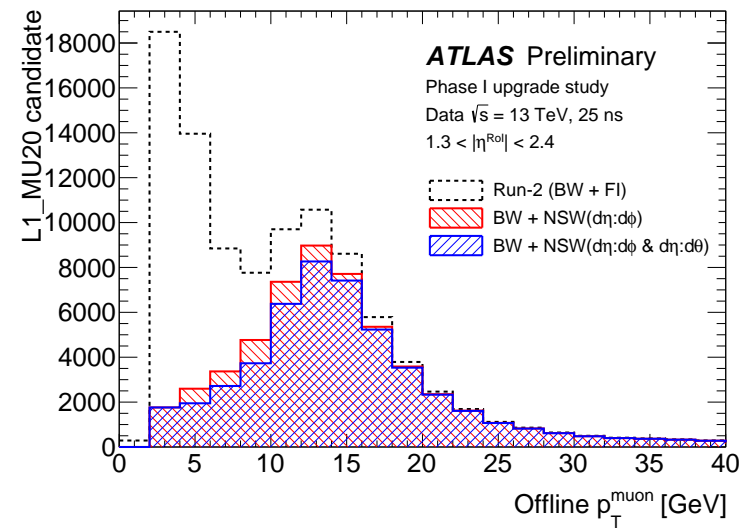

Figure 3: The number of muon candidates in L1_MU20 [6]. In the low $p_{\mathrm{T}}$ regions, the muon candidates are rejected by the inner coincidence.

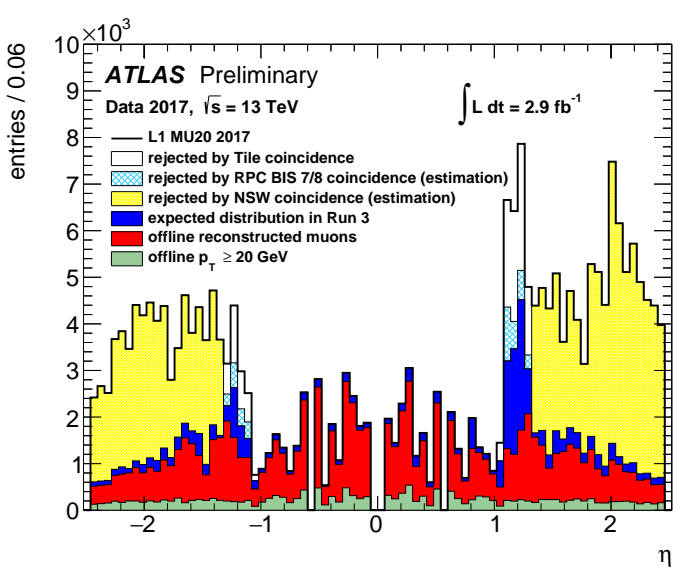

Figure 4: L1_MU20 distribution as a function of $\eta$ [6]. $90 \%$ of the fake trigger is rejected in the region covered by NSW. 


\section{Conclusion}

The level-1 endcap muon trigger will be upgraded with new detectors and electronics. Many upgrade ideas will be implemented on the FPGA of new SL. New charge identification algorithm has been developed and the performance was estimated by simulation. The charge identification accuracy is more than $98 \%$ in $p_{\mathrm{T}}<30 \mathrm{GeV}$. Also, a trigger algorithm with NSW has been developed to suppress fake triggers in the level-1 endcap muon trigger. About $45 \%$ rate reduction with negligible efficiency loss is estimated in L1_MU20 using Run 2 data.

\section{References}

[1] ATLAS Collaboration, 2008 JINST 3 S08003, https://iopscience.iop.org/article/10.1088/1748-0221/3/08/S08003/pdf

[2] ATLAS Collaboration, New Small Wheel Technical Design Report, http://cds.cern.ch/record/1552862/

[3] ATLAS Collaboration, Technical Design Report for the Phase-II Upgrade of the ATLAS Muon Spectrometer, https://cds.cern.ch/record/2285580/files/ATLAS-TDR-026.pdf

[4] ATLAS Collaboration, Proposal of upgrade of the ATLAS muon trigger in the barrel-endcap transition region with RPCs, https://pos.sissa.it/213/117/pdf

[5] HL-LHC High Luminosity Large Hadron Collider: The HLLHC project., [Online].Available:https://hilumilhc.web.cern.ch/about/hl-lhc-project

[6] ATLAS Collaboration, L1 Muon Trigger Public Results, https://twiki.cern.ch/twiki/bin/view/AtlasPublic/L1MuonTriggerPublicResults 